\title{
Lack of consistency in the associations of Helicobacter pylori seropositivity with $S e$ and $L e$ polymorphisms among Japanese
}

\author{
Nobuyuki Hamajima ${ }^{1}$, Atsuko Shibata 2 , Yuzuru Ikehara ${ }^{3}$, Nobuyuki Katsuda ${ }^{4}$, Sayomi Mori ${ }^{5}$, Hidemi Ito ${ }^{1,6}$, \\ Keitaro Matsuo ${ }^{1}$, Kazuo Tajima ${ }^{1}$, and Suketami Tominaga ${ }^{7}$ \\ ${ }^{1}$ Division of Epidemiology and Prevention, Aichi Cancer Center Research Institute, 1-1 Kanokoden, Chikusa-ku, Nagoya 464-8681, Japan \\ ${ }^{2}$ Department of Health Research and Policy, Stanford University School of Medicine, Stanford, CA, USA \\ ${ }^{3}$ Division of Oncological Pathology, Aichi Cancer Center Research Institute, Nagoya, Japan \\ ${ }^{4}$ Kita Health Center of Nagoya Municipal Government, Nagoya, Japan \\ ${ }^{5}$ Department of Hospital Laboratory, Aichi Cancer Center Hospital, Nagoya, Japan \\ ${ }^{6}$ Second Department of Internal Medicine, Nagoya City University School of Medicine, Nagoya, Japan \\ ${ }^{7}$ President of Aichi Cancer Center, Nagoya, Japan
}

\begin{abstract}
Background. In our previous study, a marked association between Helicobacter pylori seropositivity and functional polymorphisms of the secretor and Lewis genes (odds ratio [OR], 0.32; 95\% confidence interval [CI], $0.14-0.70$ for se/se genotype relative to $\mathrm{Se} / \mathrm{Se}$ genotype; and $\mathrm{OR}, 10.3$; 95\% CI, 3.1633.8 for high-risk group defined by the combination of $S e$ and $L e$ relative to low-risk group) had been observed for 239 non-cancer Japanese outpatients of the gastroenterology clinic (OGC) undergoing gastroscopy at Aichi Cancer Center Hospital.

Methods. The present study was a confirmatory study to examine the association for 679 first-visit outpatients (FVO) of all clinics at the same cancer hospital and for 465 health checkup examinees (HCE) in the same city.

Results. The associations between $H$. pylori seropositivity and the $S e$ and $L e$ genotypes were nonsignificant or even in the opposite direction among the FVO (OR, 1.52; 95\% CI, 1.00-2.32 for se/se genotype relative to $\mathrm{Se} / \mathrm{Se}$ genotype; and $\mathrm{OR}, 0.77 ; 95 \% \mathrm{CI}, \mathbf{0 . 4 3 - 1 . 4 0}$ for the high-risk group defined similarly to the previous study), and among the HCE (OR, 1.25; 95\% CI, 0.75-2.07; and OR, 1.07; 95\% CI, 0.50-2.26, respectively). The discrepancy between the previous and present results was not explained by the difference in the distributions of age, sex, smoking, and $H$. pylori seroprevalence.

Conclusion. Even in the same ethnic group, different sources of subjects may demonstrate inconsistent findings due to an unidentified effect modification. Inconsistent findings have rarely been reported by the same research group, but they are very important to understand the whole picture of the association under study.
\end{abstract}

Key word Helicobacter pylori $\cdot$ Polymorphisms · secretor gene - Lewis gene $\cdot$ PCR-CTPP

Offprint requests to: N. Hamajima

Received: May 9, 2002 / Accepted: July 2, 2002

\section{Introduction}

It is evident that Helicobacter pylori infection is related to several digestive diseases, including stomach cancer [1-3]. Acquisition of infection seems to occur primarily in childhood through the oral-oral or fecal-oral route, mainly from the family [4-6]. There is no doubt that environmental factors, especially sanitary conditions, determine the infection rate in the population. However, some individuals do not develop persistent infection even under the same poor sanitary condition, suggesting that host factors play a role in the infection and its maintenance. In addition, a twin study demonstrated that monozygous twins had a higher concordant rate of $H$. pylori infection than dizygous twins [7], indicating the importance of genetic traits.

Until recently, the information on genetic factors of the host and $H$. pylori infection was very limited; only HLA genotype and ABO blood type had been examined in relation to $H$. pylori infection [8-10]. In the past year, significant associations between $H$. pylori seropositivity and DNA sequence variants of the host were reported for the following genes; secretor (Se or FUT2) and Lewis (Le or FUT3) [11], interleukin $1 B(I L-1 B)$ [12,13], myeloperoxidase (MPO) [14], and tumor necrosis factor- $A$ (TNF-A) [15]. The Se and Le genes encode the enzymes related to the synthesis of $\mathrm{H}$ type I (type $\mathrm{O}$ substance of ABO blood type) and Lewis b (Le $\left.{ }^{\mathrm{b}}\right)$ antigens, respectively. These gene products are the ligands for blood-group antigen-binding adhesin (BabA) of $H$. pylori. The other three genes encode proinflammatory cytokines and enzymes, which could be attributed to a condition of the persistent $H$. pylori infection.

Concerning $S e$ and Le polymorphisms, the biological background for the significant association seems clear. BabA on the surface of the H. pylori membrane combines $\mathrm{H}$ type $\mathrm{I}$ and $\mathrm{Le}^{\mathrm{b}}$ expressed on the gastric epithe- 
lial cells [16-18]. The attachment of $H$. pylori to the epithelium causes a series of pathogenic processes in the gastric mucosa [19]. $\mathrm{H}$ type $\mathrm{I}$ is synthesized from the precursor by the Se enzyme, and then converted to $\mathrm{Le}^{\mathrm{b}}$ by the Le enzyme. The Le enzyme also metabolizes the precursor of $\mathrm{H}$ type I to Lewis a $\left(\mathrm{Le}^{\mathrm{a}}\right)$ antigen [20]. Low activity of Se enzyme and high activity of Le enzyme is considered to prevent the synthesis of $\mathrm{H}$ type $\mathrm{I}$ and $\mathrm{Le}^{\mathrm{b}}$, which may result in a reduced chance of $H$. pylori infection. It has been demonstrated that the sej and se5 alleles of the Se gene and the le1 and le2 alleles of the $L e$ gene have negligible or no functional activity [21-23]. In a previous study [11], we reported that low seropositivity of anti- $H$. pylori IgG antibody was associated with low expression alleles of $\mathrm{Se}$ and high expression alleles of $L e$.

Consistency, defined as the repeated observation of an association in different populations under different circumstances, is one of the criteria for establishing a causal association. It is not uncommon that epidemiologic studies of genetic polymorphisms show inconsistent results, possibly due to effect modification by genetic background and/or lifestyle. Therefore, we conducted confirmatory studies to examine whether the previously observed association of $S e$ and Le genotypes with $H$. pylori seropositivity [11] could be replicated in different study populations.

\section{Subjects and methods}

\section{Study subjects}

The subjects were sampled from first-visit outpatients of Aichi Cancer Center Hospital who participated in the Hospital-based Epidemiologic Research Program at Aichi Cancer Center - II (HERPACC-II) [24] in 2001, and from health checkup examinees who received a health checkup provided by Nagoya Municipal Government in 2000.

At the Aichi Cancer Center Hospital, approximately $20 \%$ of first-visit outpatients had a cancer, and about $60 \%$ of the first-visit outpatients donated $7 \mathrm{ml}$ of peripheral blood after they had provided written informed consent for genetic polymorphism research [24]. The population enrolled in the study consisted of 679 firstvisit outpatients who agreed to the blood donation (FVO group).

The other subject group consisted of residents of a western part of Nagoya City. Written informed consent for anonymous use of the residual blood for research purposes was obtained after blood was drawn for a health checkup. Typically, about $2 \mathrm{ml}$ of blood was left after the routine tests for health checkup. Of 489 examinees invited to participate in the study, 468 (95.7\%) agreed to provide their residual blood for genotyping and other laboratory assays. Residual blood samples from 3 subjects were not available for DNA extraction [25]. The remaining 465 health checkup examinees (HCE group) were included in this study.

\section{Laboratory tests}

Peripheral blood of the FVO subjects at Aichi Cancer Center Hospital was separated into buffy coat and plasma on the day of blood sampling. The residual blood of the HCE subjects was similarly separated, 3 days after blood sampling. The buffy coat and plasma were stored at $-40{ }^{\circ} \mathrm{C}$ after the separation. DNA was extracted from the buffy coat with a QIAamp DNA Blood Mini Kit (QIAGEN, Valencia, CA, USA). The plasma was used for an anti-H. pylori IgG antibody test ("HM-CAP", Enteric Products, Westbury, NY, USA). An enzyme-linked immunosorbent assay (ELISA) value of 2.3 or over was regarded as $H$. pylori infectionpositive [26]. The antibody test was conducted by SRL (Tokyo, Japan).

The Se gene has six alleles; Se1 (357C, 385A, 571C, and $628 C), S e 2(357 T, 385 A, 571 C$, and 628C), sej (357T, $385 T, 571 C$, and $628 C)$, se3 $(357 C, 385 A, 571 T$, and $628 C$ ), se4 (357C, 385A, 571C, and 628T), and se5 (conversion with pseudogene). Because both $\mathrm{Se} 1$ and $\mathrm{Se} 2$ have full enzyme activity, and se 3 and se 4 are very rare ( $0 \%$ out of 800 alleles) for Japanese [20], the genotyping was conducted to distinguish A385T for sej and the pseudogene for se5 from $\mathrm{Se}$ ( $\mathrm{Sel}$ and $\mathrm{Se} 2$ ) by means of a related duplex polymerase chain reaction with confronting two-pair primers (PCR-CTPP) method $[27,28]$. The primers were as follows; se5 F0, 5'-TTT CAC TGC CAC CAG CAC CTG; Se385 F1, 5'-ATC AAA GGC ACT GGG ACC CAG; Se385 R1, 5' -GGA CGT ACT CCC CCG GGA T; sej F2, 5'-TGG AGG AGG AAT ACC GCC ACT; sej R2, 5'-GTC CCC TCG GCG AAC ATG G. The underlined letters are the bases for the A385T polymorphism, and the primer se5F0 was set for the pseudogene. Genomic DNA (30-100 ng) was used for each $25-\mu l$ reaction mixture, $0.18 \mathrm{mM}$ dNTPs, $12.5 \mathrm{pmol}$ each of the five primers, $2.5 \mu \mathrm{l}$ glycerol, 0.5 units of AmpliTaq Gold, $2.5 \mu \mathrm{l}$ GeneAmp $10 \times$ PCR buffer containing $15 \mathrm{mM} \mathrm{MgCl}_{2}$ (Perkin-Elmer, Foster City, CA, USA). PCR conditions were $10 \mathrm{~min}$ of initial denaturation at $95^{\circ} \mathrm{C}$, followed by 35 cycles of $1 \mathrm{~min}$ at $95^{\circ} \mathrm{C}, 1 \mathrm{~min}$ at $61^{\circ} \mathrm{C}$, and $1 \mathrm{~min}$ at $72^{\circ} \mathrm{C}$, and a 5-min final extension at $72^{\circ} \mathrm{C}$. Amplified DNA was visualized on a $2 \%$ agarose gel containing ethidium bromide. The amplified band with $284 \mathrm{bp}$, $216 \mathrm{bp}$, and $353 \mathrm{bp}$ represents the $S e$ allele (385A), sej $(385 T)$, and se5 allele, respectively, while a common band of $460 \mathrm{bp}$ appeared for the $S e$ and $s e j$ alleles, as demonstrated in Fig. 1a. 


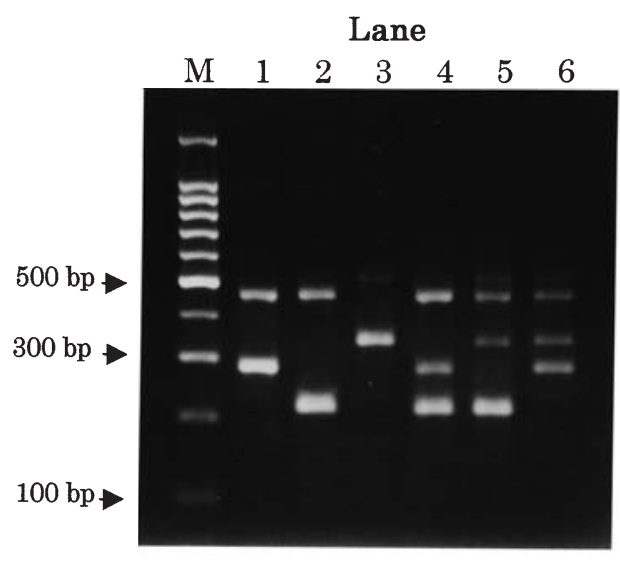

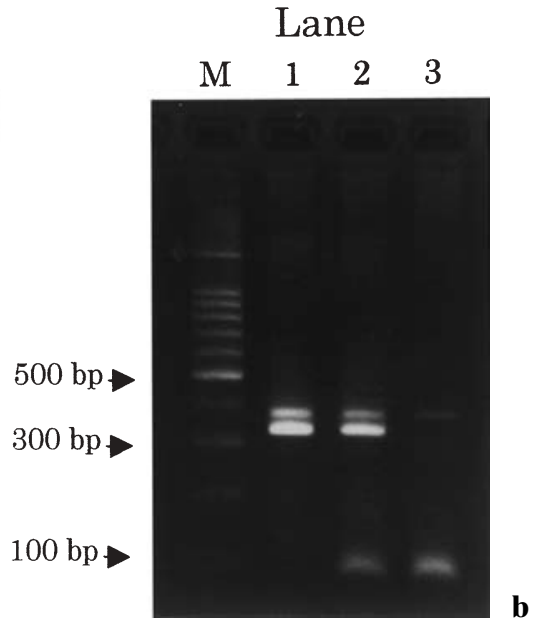

Fig. 1a,b. Representative examples of agarose gel electrophoresis. a $\mathrm{Se}$ A385T and conversion type (se5) polymorphisms. Lane $M, 100$-bp ladder marker, lane 1, A/A (Se/Se); lane 2, T/T (se/se); lane 3, se5se 5 (se/se); lane 4, A/T ( Se/se); lane 5, T/se5 (se/se); and lane 6, A/se5 ( $\mathrm{Se} / \mathrm{se}$ ). b Le T59G polymorphism. Lane $M, 100$-bp ladder marker; lane $1, T / T$ (Le/Le); lane 2, T/G (Le/le); and lane 3, G/G (le/le)
The Le gene has four alleles; Le $(59 T, 508 G$, and $1067 T)$, le1 (59G, 508A, and 1067T), le2 (59G, 508G, and $1067 A$ ), and $l e 3(59 G, 508 G$, and $1067 T)$. Because le 3 is very rare $(0.5 \%$ out of 800 alleles) for Japanese [20], T59G was genotyped to distinguish le1 and le2 from $L e$ by PCR-CTPP. The primers were: F1, $5^{\prime}$-CCA TGG ATC CCC TGG GTG; R1, 5'-CCA CCA GCA GCT GAA ATA GCC; F2, 5'-CGC TGT CTG GCC GCA CT; and R2, 5'-GAA GGT GGG AGG CGT GAC TTA. Genomic DNA (30-100ng) was used for each $25-\mu \mathrm{l}$ reaction with $0.18 \mathrm{mM}$ dNTPs, $25 \mathrm{pmol}$ each of the primers, 0.5 units of AmpliTaq Gold, and $2.5 \mu \mathrm{l}$ GeneAmp 10× PCR buffer containing $15 \mathrm{mM} \mathrm{MgCl}_{2}$ (Perkin-Elmer). The PCR conditions were same as those for the Se genotyping, except for annealing at $66^{\circ} \mathrm{C}$. Amplified were a 329-bp band for the $T$ allele ( $L e$ ) and an 81-bp band for the $G$ allele (le1 and le2), while a common band of $373 \mathrm{bp}$ appeared for both alleles (Fig. 1b).

The present study had been approved by the Ethics Committee at Aichi Cancer Center before the study started (Ethics Committee Approval Numbers 41-2 and 11-12).

\section{Statistical analysis}

An unconditional logistic model was applied for estimating odds ratios (ORs), using a computer program (STATA version 7; STATA, College Station, TX, USA). ORs were adjusted for age as a dichotomous variable ( $<50$ years vs $\geq 50$ years). Goodness-of-fit of genotype distribution to Hardy-Weinberg equilibrium was examined by the "genhwi" procedure of STATA.

\section{Results}

Table 1 shows anti-H. pylori IgG seropositivity according to sex and age group for FVO and HCE subjects. The ages ranged from 18 to 79 years for the FVO and from 32 to 85 years for the HCE subjects. The seropositivity increased with age, and leveled off around age 50 years for both groups of subjects. The age-specific rate for men was slightly higher or equal to that for women, except for the 18-to-29-year-olds in the FVO group and the 30-to-39-year-olds in the HCE group.

The percentages of self-reported current smokers at study entry were $35.9 \%$ in men and $13.2 \%$ in women in the FVO group, and $38.6 \%$ in men and $5.6 \%$ in women in the HCE group. The seroprevalence of anti-H. pylori antibody in the male $\mathrm{FVO}$ group was $59.3 \%$ for current smokers $(n=113), 61.0 \%$ for former smokers $(n=$ $136)$, and 62.1 for never smokers $(n=66)$; and in the female FVO group, the seroprevalences were $45.8 \%$ $(n=48), 53.3 \%(n=30)$, and $46.7 \%(n=285)$, respectively. The corresponding prevalences in the HCE group were $59.2 \%(n=49), 85.7 \%(n=7)$, and $69.9 \%$ $(n=71)$ for men; and $42.1 \%(n=19) ; 20.0 \%(n=5)$, and $51.6 \%(n=314)$ for women. The difference in $H$. pylori seroprevalence between current smokers and never smokers was not statistically significant for either sex according to subject group by a $\chi^{2}$ test.

The $S e$ genotype was determined for all subjects, except for $1 \mathrm{HCE}$ subject, and the Le genotype was determined for $663(97.6 \%)$ of the 679 FVO subjects and 424 $(91.2 \%)$ of the $465 \mathrm{HCE}$ subjects. The genotype frequencies of each polymorphism are shown in Table 2 . The genotype distribution was in Hardy-Weinberg equilibrium $\left(\chi^{2}=0.77 ; P=0.38\right.$ for $S e$, and $\chi^{2}=2.19$; $P=0.14$ for Le among the outpatients; and $\chi^{2}=1.45$; 
Table 1. Seroprevalence of anti-Helicobacter pylori antibody by study population, age, and sex

\begin{tabular}{|c|c|c|c|c|c|c|c|c|}
\hline \multirow{3}{*}{$\begin{array}{l}\text { Age } \\
\text { (years) }\end{array}$} & \multicolumn{4}{|c|}{ First-visit outpatients (FVO) } & \multicolumn{4}{|c|}{ Health checkup examinees (HCE) } \\
\hline & \multicolumn{2}{|r|}{ Male } & \multicolumn{2}{|c|}{ Female } & \multicolumn{2}{|r|}{ Male } & \multicolumn{2}{|c|}{ Female } \\
\hline & $n$ & H. pylori $+\%^{\mathrm{a}}$ & $n$ & H. pylori $+\%$ & $n$ & H. pylori $+\%$ & $n$ & H. pylori $+\%$ \\
\hline $18-29$ & 14 & 7.1 & 25 & 8.0 & 0 & - & 0 & - \\
\hline $30-39$ & 24 & 33.3 & 69 & 27.5 & 9 & 11.1 & 40 & 17.5 \\
\hline $40-49$ & 35 & 54.3 & 66 & 47.0 & 7 & 42.9 & 51 & 39.2 \\
\hline $50-59$ & 97 & 66.0 & 113 & 52.2 & 10 & 60.0 & 97 & 58.8 \\
\hline $60-69$ & 91 & 69.2 & 60 & 68.3 & 65 & 75.4 & 116 & 56.9 \\
\hline $70-79$ & 54 & 66.7 & 31 & 61.3 & 32 & 71.9 & 30 & 63.3 \\
\hline $80-85$ & 0 & - & 0 & - & 4 & 50.0 & 4 & 50.0 \\
\hline Total & 315 & 60.6 & 364 & 47.0 & 127 & 66.1 & 338 & 50.6 \\
\hline
\end{tabular}

${ }^{\mathrm{a}}$ H. pylori $+\%$, Percentage of anti-H. pylori antibody-positive subjects

Table 2. Genotype distributions of Secretor and Lewis genes

\begin{tabular}{|c|c|c|c|c|}
\hline \multirow[b]{2}{*}{ Genotype } & \multicolumn{2}{|c|}{ First-visit outpatients (FVO) } & \multicolumn{2}{|c|}{ Health checkup examinees (HCE) } \\
\hline & $n(\%)$ & H. pylori $+{ }^{\mathrm{a}}(H$. pylori $+\%)$ & $n(\%)$ & H. pylori+ $($ H. pylori $+\%)$ \\
\hline \multicolumn{5}{|c|}{ Secretor gene } \\
\hline Sese & $170(25.0)$ & $79(46.5)$ & $139(30.0)$ & $69(49.6)$ \\
\hline Sese & $328(48.3)$ & $180(54.9)$ & $218(47.0)$ & $126(57.8)$ \\
\hline sese & $181(26.7)$ & $103(56.9)$ & $107(23.1)$ & $59(55.1)$ \\
\hline Total & $679(100)$ & $362(53.3)$ & $464(100)$ & $254(54.7)$ \\
\hline \multicolumn{5}{|l|}{ Lewis gene } \\
\hline LeLe & $353(53.2)$ & $189(53.5)$ & $235(55.7)$ & $126(53.4)$ \\
\hline Lele & $251(37.9)$ & $132(52.6)$ & $155(36.6)$ & $87(56.1)$ \\
\hline lele & $59(8.9)$ & $36(61.0)$ & $33(7.8)$ & $20(60.6)$ \\
\hline Total & $663(100)$ & $357(53.8)$ & $424(100)$ & $233(55.0)$ \\
\hline
\end{tabular}

${ }^{a}$ H. pylori+, Anti-H. pylori antibody-positive

$P=0.23$ for $S e$, and $\chi^{2}=1.12 ; P=0.29$ for $L e$ among the examinees). When both sexes were combined, a $\chi^{2}$ test for 2 (seronegative vs seropositive) by 3 (genotypes) showed no statistically significant associations between the $H$. pylori seropositivity and the genotypes of $S e$ and Le for either group, although the seroprevalence was higher among individuals with sese or lele than among those with $\mathrm{SeSe}$ or LeLe, respectively.

Table 3 shows the sex-and-age-adjusted ORs for $H$. pylori seropositivity by study population. The OR for FVO subjects with the se allele (Sese or sese) was significantly higher than that for those with SeSe, and the adjusted OR for HCE subjects with Sese was marginally significant (95\% confidence interval, 1.00-2.46). The Le genotype showed no significant association with seropositivity for either study population.

We also estimated ORs for $H$. pylori seropositivity according to risk groups created based on the results of our previous study [11]; low-risk (sese \& LeLe), moderate risk (combinations other than low- or high-risk groups), and high-risk (SeSe \& lele, SeSe \& Lele, and
Sese \& lele) groups. In the present study, no statistically significant association was observed between the combined genotype risk group and $H$. pylori seropositivity.

\section{Discussion}

This study did not reproduced the association of $H$. pylori seropositivity with the $S e$ and Le polymorphisms observed in the preceding study of 239 outpatients (118 males and 121 females) who underwent gastroscopy at the Gastroenterology Clinic (OGC) of Aichi Cancer Center Hospital [11]. The subjects of the previous study included 95 (39.7\% of 239) participants who stated that they were taking medication for diseases (not confirmed by their medical records); 23 subjects were taking medication for gastric/duodenal ulcer, another 23 for socalled gastritis, and 59 for miscellaneous diseases or conditions (i.e., some subjects were taking medication for more than one disease; no. of diseases $=105)$. The H. pylori seropositivity was $72.1 \%$ for $\mathrm{SeSe}, 64.6 \%$ for 
Table 3. Odds ratios (ORs) and $95 \%$ confidence intervals (95\% CIs) for H. pylori seropositivity

\begin{tabular}{|c|c|c|c|c|}
\hline \multirow[b]{2}{*}{ Genotype } & \multicolumn{2}{|c|}{ First-visit outpatients (FVO) } & \multicolumn{2}{|c|}{ Health checkup examinees (HCE) } \\
\hline & $\operatorname{cOR}^{\mathrm{a}}(95 \% \mathrm{CI})$ & $\mathrm{aOR}^{\mathrm{b}}(95 \% \mathrm{CI})$ & $\operatorname{cOR}^{\mathrm{a}}(95 \% \mathrm{CI})$ & $\mathrm{aOR}^{\mathrm{b}}(95 \% \mathrm{CI})$ \\
\hline \multicolumn{5}{|l|}{ Secretor gene } \\
\hline $\mathrm{SeSe}$ & 1 (Reference) & 1 (Reference) & 1 (Reference) & 1 (Reference) \\
\hline Sese & $1.40(0.97-2.03)$ & $1.51(1.02-2.22)$ & $1.39(0.91-2.13)$ & $1.57(1.00-2.46)$ \\
\hline sese & $1.52(1.00-2.32)$ & $1.50(0.97-2.33)$ & $1.25(0.75-2.07)$ & $1.29(0.76-2.19)$ \\
\hline Sese/sese & $1.44(1.02-2.04)$ & $1.50(1.05-2.16)$ & $1.34(0.90-2.00)$ & $1.47(0.97-2.23)$ \\
\hline \multicolumn{5}{|l|}{ Lewis gene } \\
\hline LeLe & 1 (Reference) & 1 (Reference) & $1 \quad$ (Reference) & 1 (Reference) \\
\hline Lele & $0.96(0.70-1.33)$ & $0.98(0.70-1.37)$ & $1.12(0.74-1.68)$ & $1.06(0.69-1.63)$ \\
\hline lele & $1.36(0.77-2.39)$ & $1.31(0.73-2.37)$ & $1.34(0.64-2.83)$ & $1.40(0.63-3.07)$ \\
\hline Lele/lele & $1.03(0.76-1.39)$ & $1.03(0.75-1.42)$ & $1.15(0.78-1.70)$ & $1.11(0.74-1.67)$ \\
\hline \multicolumn{5}{|c|}{ Combined risk group ${ }^{c}$} \\
\hline Low & 1 (Reference) & 1 (Reference) & 1 (Reference) & 1 (Reference) \\
\hline Moderate & $0.71(0.45-1.11)$ & $0.79(0.49-1.28)$ & $1.28(0.71-2.34)$ & $1.50(0.81-2.81)$ \\
\hline High & $0.77(0.43-1.40)$ & $0.77(0.43-1.40)$ & $1.07(0.50-2.26)$ & $1.07(0.49-2.34)$ \\
\hline
\end{tabular}

${ }^{a}$ Crude OR

${ }^{\mathrm{b}}$ Sex-and-age-adjusted OR by dichotomous variables (male vs female and age $<50$ years vs $\geq 50$ years)

${ }^{\mathrm{c}}$ Based on the results of a previous study [11]. Low-risk, sese \& LeLe; high-risk, SeSe \& lele, SeSe \& Lele, and Sese \& lele; moderate risk, the other combinations

Sese, and $45.1 \%$ for sese, and $55.6 \%$ for LeLe, $68.4 \%$ for LeLe, and $76.5 \%$ for lele. When the genotypes were combined, the sex-and-age-adjusted OR relative to individuals with sese \& LeLe (low-risk group) was 10.21 (95\% confidence interval, 2.98-34.96) for individuals with $\mathrm{SeSe} \&$ lele, $\mathrm{SeSe} \& \mathrm{LeLe}$, or Sese \& lele (high-risk group) and 3.34 (1.36-8.20) for the remaining individuals (moderate risk group). Even when the 23 ulcer patients and 23 gastritis patients were excluded, a strong association was observed (the ORs became larger). The observed association was consistent with a biologically plausible hypothesis described in the "Introduction" in this article.

The lack of or the opposite associations observed for individuals in the same geographic area as those in the previous study [11] were a surprise to us. We expected that such associations with genetic polymorphisms would be consistent within one ethnic group, especially in the same geographic area. For example, we observed consistent associations between $H$. pylori seropositivity and the $I L-1 B$ C-31T polymorphism among Japanese in three datasets $[12,13,29]$, between the Dopamine Receptor $D 2$ polymorphism and smoking habit in two datasets $[30,31]$, and between the $I L-1 B$ C-31T polymorphism and smoking habit in two datasets [25].

In epidemiologic studies of genetic polymorphisms and disease risk, inconsistent results are not rare among ethnically and/or geographically diverse groups even after eliminating the possibilities of various biases. Confounding by race/ethnicity, also known as population stratification, is a well-recognized phenomenon observed in a study that includes subjects from different ethnic groups. Inconsistent findings could result from other factors, such as random errors, unknown effect modification, dilution of the association strength, and their combinations. We discuss below these potential explanations for the inconsistency observed among three studies conducted in Aichi, Japan.

The first potential explanation is random error. Our previous study for OGC had 239 subjects, including 90 seronegative individuals. Seventeen of 61 subjects with $\mathrm{SeSe}$ and 4 of 17 subjects with lele were seronegative. The main purpose of the present study was to confirm the promising finding of the previous study [11], using 527 seronegative and 617 seropositive subjects (in total 1144) sampled from the FVO and HCE groups. Because the $95 \%$ confidence interval of the OR for the high-risk group of OGC did not overlap with those observed in the present study, a random error may be not the cause of the inconsistent results. The present study with larger sample size should provide statistically more stable results than our previous study of OGC, if there were neither effect modifiers nor different degrees of dilution.

The second possibility is unknown effect modification, or interaction between risk factors (genetic or environmental). Gene-gene interactions and geneenvironment interactions have been documented for several diseases [32-35]. We observed that smoking habit was not associated with $H$. pylori seropositivity in FVO [29] and HCE subjects [13], while it was associated with the seropositivity in OGC subjects [12]. Although there is a controversy concerning the association between $H$. pylori infection and smoking habit [36], the inconsistent results on smoking seem to be due to an effect modification; the association tends to be stronger 
for patients with gastrointestinal complaints than for healthy residents; stronger in high-prevalence than in low-prevalence areas; and stronger in populations with a high smoking prevalence than in populations with a low smoking prevalence [37]. In this study, the sese genotype percentages $(26.7 \%$ in the 679 FVO subjects and $23.1 \%$ in the $464 \mathrm{HCE}$ subjects) were similar to or slightly higher than those observed for Japanese in other areas $(24.8 \%$ of 141 subjects [22], $21.3 \%$ of 231 subjects [11], and 18.5 of 400 subjects [20]), and the lele genotype percentages $(8.9 \%$ of $663 \mathrm{FVO}$ subjects and $7.8 \%$ of $424 \mathrm{HCE}$ subjects) were also similar to those in other studies $(10.0 \%$ of 400 subjects [20] and $7.1 \%$ of 239 subjects [11]). The association between $H$. pylori seropositivity and $\mathrm{Se}$ and $\mathrm{Le}$ polymorphisms, however, may be modified by unknown factors.

Dilution of the association occurs when subjects whose outcome under study is not associated with risk factors of interest (e.g., those whose seropositivity is not influenced by $S e$ and $L e$ genotypes) are mixed with subjects for whom the outcome and risk factors are associated. An overall OR for the whole study population would become closer to unity (i.e., null association) with an increased proportion of subjects in the former group.

In the present study, there was no information on the mixture of individuals whose $H$. pylori seropositivity was not associated with the $S e$ and $L e$ genotypes. However, it is possible that $H$. pylori with BabA might be more prevalent in OGC than in FVO and HCE subjects. Because $\mathrm{H}$ type $\mathrm{I}$ and $\mathrm{Le}^{\mathrm{b}}$ antigens play a role in the attachment of BabA-positive H. pylori to gastric epithelial cells $[18,19]$, the $S e$ and $L e$ genotypes do not influence the susceptibility for those with BabA-negative $H$. pylori. If BabA-positive $H$. pylori was associated with the hospital visit of $H$. pylori infected individuals due to severe gastritis [38], the marked association observed only for OGC would be explained. In Japan, the $B a b A 2$ gene encoding BabA was reportedly highly prevalent ( $84.9 \%$ of 152 strains) in patients who underwent gastroscopy [39], but no data are available for healthy residents. We could not examine for this hypothetical possibility, because $H$. pylori samples from our subjects were not available.

In summary, the association between $H$. pylori seropositivity and functional polymorphisms of $S e$ and $L e$ was not reproduced in the present study of 679 first-visit outpatients and 465 health checkup examinees. Because the association is biologically plausible, the inconsistent findings observed for the same ethnic group in the same geographic area may be due to unknown effect modification and/or dilution of association strength, although they could be due to other types of bias. Like other inconsistent findings observed for polymorphisms and disease risk, potential effect modifiers remain to be identified. Inconsistent findings are often unreported, but we believe it is important to report them to reduce the chance of reaching wrong conclusions due to publication bias.

Acknowledgments This work was supported in part by a Grant-in-Aid for the Second Term Comprehensive 10Year Strategy for Cancer Control from the Ministry of Health, Labour and Welfare of Japan. The authors are grateful to Ms. Naomi Takeuchi for genotyping.

\section{References}

1. Labenz J, Borsch G. Evidence for the essential role of Helicobacter pylori in gastric ulcer disease. Gut 1994;35:19-22.

2. Munoz N. Is Helicobacter pylori a cause of gastric cancer? An appraisal of the seroepidemiological evidence. Cancer Epidemiol Biomarkers Prev 1994;3:445-41.

3. Dunn BE, Cohen H, Blaser MJ. Helicobacter pylori. Clin Microbiol Rev 1997;10:720-41.

4. Goodman K, Correa P. The transmission of Helicobacter pylori. A critical review of the evidence. Int J Epidemiol 1995;24:875-87.

5. Brown LM. Helicobacter pylori: epidemiology and routes of transmission. Epidemiol Rev 2000;22:283-97.

6. Tindberg Y, Bengtsson C, Granath F, Blennow M, Nyrén O, Granström. Helicobacter pylori infection in Swedish school children: lack of evidence of child-to-child transmission outside the family. Gastroenterology 2001;121:310-6.

7. Malaty HM, Engstrand L, Pedersen NL, Graham DY. Helicobacter pylori infection: genetic and environmental influence. A study of twins. Ann Intern Med 1994;129:982-6.

8. Niv Y, Fraser G, Delpre G, Neeman A, Leiser A, Samra Z, et al. Helicobacter pylori and blood groups. Am J Gastroenterol 1996; 91:101-4.

9. Go MF. What are the host factors that place an individual at risk for Helicobacter pylori - associated disease? Gastroenterology 1997;113:S15-S20.

10. Nguyen TN, Barkun AN, Fallone CA. Host determinants of Helicobacter pylori infection and its clinical outcome. Helicobacter 1999;4:185-97.

11. Ikehara Y, Nishihara S, Yasutomi H, Kitamura T, Matsuo K, Shimizu N, et al. Polymorphisms of the two fucosyltransferase genes (Lewis and Secretor genes) involving type I Lewis antigens are associated with the presence of anti-Helicobacter pylori IgG antibody. Cancer Epidemiol Biomarkers Prev 2001;10:9717.

12. Hamajima N, Matsuo K, Saito T, Tajima K, Okuma K, Yamao K, et al. Interleukin 1 polymorphisms, lifestyle factors, and Helicobacter pylori infection. Jpn J Cancer Res 2001;92:383-9.

13. Katsuda N, Hamajima N, Matsuo K, Saito T, Ito LS, Inoue M, et al. Association between the interleukin 1B (C-31T) polymorphism and Helicobacter pylori infection in health checkup examinees. Nippon Koshu Eisei Zasshi (Jpn J Public Health) 2001;48:604-12.

14. Hamajima N, Matsuo K, Suzuki T, Nakamura T, Matsuura A, Tajima K, et al. Low expression myeloperoxidase genotype negatively associated with Helicobacter pylori infection. Jpn J Cancer Res 2001;92:488-93.

15. Yea SS, Yang Y-I, Jang WH, Lee Y-J, Bae H-S, Paik KH. Association between TNF- $\alpha$ promoter polymorphism and Helicobacter pylori cagA subtype infection. J Clin Pathol 2001;54: 703-6.

16. Boren T, Falk P, Roth KA, Larson G, Normark S. Attachment of Helicobacter pylori to human gastric epithelium mediated by blood group antigens. Science 1993;262:1892-5. 
17. Ilver D, Arnqvist A, Ögren J, Frick I-M, Kersulyte D, Incecik ET, et al. Helicobacter pylori adhesin binding fucosylated histoblood group antigens revealed by retagging. Science 1998;279: 373-7.

18. Gerhard M, Lehn N, Neumayer N, Borén T, Rad R, Schepp W, et al. Clinical relevance of the Helicobacter pylori gene for bloodgroup antigen-binding adhesin. Proc Natl Acad Sci USA 1999;96: 12778-83.

19. Montecucco C, Rappuoli R. Living dangerously: how Helicobacter pylori survives in the human stomach. Nat Rev 2001;2:45766.

20. Narimatsu H, Iwasaki H, Nakayama F, Ikehara $Y$, Kudo T, Nishihara S, et al. Lewis and secretor gene dosages affect CA19-9 and DU-PAN-2 serum levels in normal individuals and colorectal cancer patients. Cancer Res 1998;58:512-18.

21. Kudo T, Iwasaki H, Nishihara S, Shinya N, Ando T, Narimatsu I, et al. Molecular genetic analysis of the human Lewis histo-blood group system. II. Secretor gene inactivation by a novel single missense mutation A385T in Japanese nonsecretor individuals. J Biol Chem 1996;271:9830-7.

22. Koda Y, Soejima M, Liu Y, Kimura H. Molecular basis for secretor type alpha(1,2)-fucosyltransferase gene deficiency in a Japanese population: a fusion gene generated by unequal crossover responsible for the enzyme deficiency. Am J Hum Genet 1996;59:343-50.

23. Nishihara S, Narimatsu H, Iwasaki H, Yazawa S, Akamatsu S, Ando T, et al. Molecular genetic analysis of the human Lewis histo-blood group system. J Biol Chem 1994;269:29271-8.

24. Hamajima N, Matsuo K, Saito T, Hirose K, Inoue M, Takezaki T, et al. Gene-environment interaction and polymorphism studies for cancer risks in Hospital-based Epidemiologic Research Program at Aichi Cancer Center II (HERPACC-II). Asian Pacific J Cancer Prev 2001;2:99-107.

25. Hamajima N, Katsuda N, Matsuo K, Saito T, Ito LS, Ando M, et al. Smoking habit and Interleukin $1 B C-31 T$ polymorphism. J Epidemiol 2001;11:120-5.

26. Matsuo K, Hamajima N, Suzuki T, Nakamura T, Matsuura A, Kitayama K, et al. Helicobactor pylori IgG antibody test established in the United States showed a substantially lower sensitivity for Japanese population. Am J Gastroenterol 2000;95:1597-8.

27. Hamajima N, Saito T, Matsuo K, Tajima K. Competitive amplification and unspecific amplification in polymerase chain reaction with confronting two-pair primers (PCR-CTPP). J Mol Diagn 2002;4:103-7.

28. Hamajima N. PCR-CTPP: a new genotyping technique in the era of genetic epidemiology. Exp Rev Mol Diagn 2001;1:119-23.
29. Hamajima N, Ito H, Matsuo K, Tajima K, Tominaga S. Helicobacter pylori seropositivity, the interleukin $1 B$ polymorphism, and smoking among first-visit outpatients. Asian Pacific J Cancer Prev 2002;3:23-8.

30. Yoshida K, Hamajima N, Kozaki K, Saito H, Maeno K, Sugiura T, et al. Association between the dopamine D2 receptor A2/A2 genotype and smoking behavior in the Japanese. Cancer Epidemiol Biomarkers Prev 2001;10:403-5.

31. Hamajima N, Ito H, Matsuo K, Saito T, Tajima K, Ando M, et al. Association between smoking habits and dopamine receptor D2 TaqI A2 allele in Japanese males: a confirmatory study. J Epidemiol 2002;12:297-304.

32. Taylor JA, Umbach DM, Stephens E, Castranio T, Paulson D, Robertson $\mathrm{C}$, et al. The role of $N$-acetylation polymorphisms in smoking-associated bladder cancer: evidence of a gene-geneexposure three-way interaction. Cancer Res 1998;58:3603-10.

33. Mucci LA, Wedren S, Tamimi RM, Trichopoulos D, Adami HO. The role of gene-environment interaction in the aetiology of human cancer: examples from cancers of the large bowel, lung and breast. J Intern Med 2001;249:477-93.

34. Matsuo K, Hamajima N, Shinoda M, Hatooka S, Inoue $M$, Takezaki T, et al. Gene-environment interaction between an aldehyde dehydrogenase-2 (ALDH2) polymorphism and alcohol consumption for the risk of esophageal cancer. Carcinogenesis 2001;22:912-6.

35. Kumimoto H, Hamajima N, Nishizawa K, Nishimoto Y, Matsuo $\mathrm{K}$, Harada H, et al. Different susceptibility of each $L-m y c$ genotype to esophageal cancer risk factors. Jpn J Cancer Res 2001; 92:735-9.

36. Parasher G, Eastwood GL. Smoking and peptic ulcer in the Helicobacter pylori era. Eur J Gastroenterol Hepatol 2000; 12:843-53.

37. Hamajima $\mathrm{N}$, Inoue $\mathrm{M}$, Tajima $\mathrm{K}$, Tominaga S, Matsuura A, Kobayashi S, et al. Lifestyle and anti-Helicobacter pylori immunoglobulin G antibody among outpatients. Jpn J Cancer Res 1997; 88:1038-43.

38. Prinz C, Schoniger M, Rad R, Becker I, Keiditsch E, Wagenpfeil, et al. Key importance of the Helicobacter pylori adherence factor blood groups antigen binding adhesin during chronic gastric inflammation. Cancer Res 2001;61:1903-9.

39. Mizushima T, Sugiyama T, Komatsu Y, Ishizuka J, Kato M, Asaka M. Clinical relevance of the babA2 genotype of Helicobacter pylori in Japanese clinical isolates. J Clin Microbiol 2001; 39:2463-5. 